\title{
Kinematics Analysis: Number of Trials Necessary to Achieve Performance Stability during Soccer Instep Kicking
}

\author{
by \\ Mohammadtaghi Amiri-Khorasani ${ }^{1}$, Noor Azuan Abu Osman ${ }^{2}$, Ashril Yusof ${ }^{1}$
}

\begin{abstract}
The purpose of this study was to investigate the stability of kinematics responses related to stretch shortening cycle (SSC) during 10 consecutive soccer instep kicks. The kicking motions of dominant legs were captured from five experienced adult male soccer players (body height: $184.60 \pm 4.49 \mathrm{~cm}$; body mass: $80 \pm 4.24 \mathrm{~kg}$; age: $25.60 \pm 1.14$ years) using a three-dimensional infra-red high speed camera at $200 \mathrm{~Hz}$. Some important kinematic parameters include eccentric angular velocity $(A V e)$, concentric angular velocity $(A V c)$, duration of eccentric (Te), and duration of concentric (Tc) at forward and impact phases selected to analyses. The AVe result of the sixth kick, relative to the first kick, was significantly lower when compared to the other kicks (with $p \leq 0.001$ ). The AVc result of the fifth kick, relative to the first kick, was significantly lower when compared to the other kicks (with $p \leq 0.001$ ). The Te result of the fourth kick, relative to the first kick, was significantly lower when compared to the other kicks (with $p \leq 0.011$ ). The Tc result of the fifth kick, relative to the first kick, was significantly lower when compared to the other kicks ( $p \leq$ 0.029). We concluded that 5 consecutive kicks are adequate to achieve high kinematic responses related to SSC.
\end{abstract}

Key words: Angular velocity, Concentric, Eccentric, Soccer instep Kicking

\section{Introduction}

Variability is present in all human movement; thus using too few trials may not represent the individual's long-term performance (James, 2007). A single trial protocol has been suggested to be both invalid and unreliable (Bates, 1992) because of the potential inability of the single trial to represent the generalized performance. By chance, the single trial could represent an average performance but also might be atypical. Greater movement variability results in less stable data and a greater likelihood of sampling an atypical performance from the population of all possible performances. While increasing the number of trials is thought to increase perform- ance stability (James, 2007; Salo, 1997), how many trials are necessary to provide stable data?

Little information is available about the number of trials necessary to achieve performance stability for soccer instep kicking, which is the most offensive action during the game (Kellis and Katis. 2007; Amiri-Khorasani et al., 2009). In general, researchers divided soccer instep kick into three phases which include (1) preparation or backswing, (2) action or forward swing, (3) Impact or ball contact, and (4) recovery or follow-through (Lees and Nolan, 1998; Bartlett, 2006; Kellis and Katis, 2007). Each one of these phases has specific biomechanical functions. It is suggested that the latter phases depend upon the previous phase or phases (Bartlett, 2006). Therefore, the first phase plays an important role to support the

\footnotetext{
1 - Sport Centre, University of Malaya, Malaysia.

2 - Department of Biomedical Engineering, University of Malaya, Malaysia.
} 
subsequent phases in producing more force as result of stretch shortening cycle (SSC) (Manolopoulos et al., 2004; Sedano-Campo et al., 2009). It is proposed that in simulated kicking actions, the speed of the movement can be increased by up to $21 \%$ when using a SSC rather than a purely concentric muscular contraction to extend the knee joint. It has already been noted that, in the mature kicking action, the thigh is brought forward while the knee is still flexing. This action serves to stretch the extensor muscles of the thigh before they are required to shorten, so that they are able to aid in the generation of large end-point speed (Lees and Nolan, 1998).

More research studies into biomechanics of soccer kick have been published within the last decade. Therefore, new aspects of soccer kick performance are being identified, including more details regarding the three-dimensional kinematics of the movement (Levanon and Dapena, 1998; Nunome et al., 2006), joint-motion that drive the movement (Nunome et al., 2002; Nunome et al., 2006), mechanisms of soccer performance, as well as various factors which affect soccer kick biomechanics, such as age, gender, limb dominance and fatigue (Barfield, 1995; Dorge et al., 2002; Kellis and Katis, 2007). However, there are no clear results for number of trials required for accurate data analysis during research, which seems to be an important methodological consideration in the design of kicking experiments. Therefore, we investigated the number of trials necessary to obtain high kinematic responses related to SSC during 10 consecutive soccer instep kicks, and determine the decreasing rate of time and angular velocity of eccentric and concentric contraction during 10 consecutive soccer instep kick. It was thereby our aim to test the hypothesis that 10 consecutive kicks will not provide optimal kinematic responses, relative to SSC.

\section{Materials $\mathcal{E}$ methods}

Five experienced adult male soccer players (body height: $184.60 \pm 4.49 \mathrm{~cm}$; body mass: $80 \pm 4.24 \mathrm{~kg}$; age: $25.60 \pm 1.14$ years), who had no history of major lower limb injury or disease, volunteered to participate in this study. The Sport Centre Committee at university gave approval for all procedures. Subjects were required to report to the research laboratory to read and sign a medical questionnaire and an informed consent. All participants were training regularly.
As all participants preferred to kick the ball using their right leg, the right leg was considered the dominant leg. After an adequate warm up, each participant performed 10 maximal velocity instep place kicks of a stationary ball; essentially, corresponding to the penalty kick in soccer. A ball was kicked $3 \mathrm{~m}$ from a target $1 \times 1 \mathrm{~m}$ in size. With an easy target to hit, the subjects were encouraged to strike the ball as hard a possible. To minimize movement in the frontal plane, the participants were restricted to a $3 \mathrm{~m}$ straight run-up from a position directly behind the ball, at an approach angle of $0^{\circ}$. An FIFAapproved size 5 soccer ball (mass $=435 \mathrm{~g}$ ) was used for each kicking session, and its pressure was controlled throughout the trials at $700 \mathrm{hPa}$.

Six synchronized high-speed infra-red cameras (Vicon MX-F20) were used to capture limb motion at $200 \mathrm{~Hz}$. Once the cameras were positioned into the appropriate locations (performance area), the cameras were then calibrated to define their own volume origin. A motion capture software (Vicon Nexus 1.2) was used to digitize body landmarks, including the bony anatomical landmarks of the right and left anterior superior iliac spine, posterior superior iliac spine, mid-way between the posterior superior iliac spines, lateral epicondyle of knee, thigh; over the lower lateral $1 / 3$ surface of the thigh, lateral malleolus, shank; over the lower $1 / 3$ of the shank, over the second metatarsal head, and calcaneous.

The dependent variables selected were peak angular velocity of concentric contraction $(\mathrm{AVc})$, peak angular velocity of eccentric contraction (AVe), and the durations of the eccentric (Te) and concentric (Tc) phases of soccer instep kicking. These variables were selected to document an overall measure of performance and several angular measures related to the SSC in soccer instep kick. The durations of the eccentric and concentric phases were operationally defined relative to flexion motion of the knee from toe off to deepest knee flexion, and from knee extension to ball impact, respectively.

An analysis of variance (one-way ANOVA) was conducted to examine significance within variables, and Tukey's post-test was used to compare each of the above defined kinematic variables of kicking, between the 10 consecutive instep kicks. A 0.05 alpha level was used to indicate statistical significance. Test-retest reliability values for the testing order of tests ICCRs (intraclass correlation reliability) were $\geq$ 0.89 , effect size was $\geq 0.93$ and power was $\geq 0.99$. 


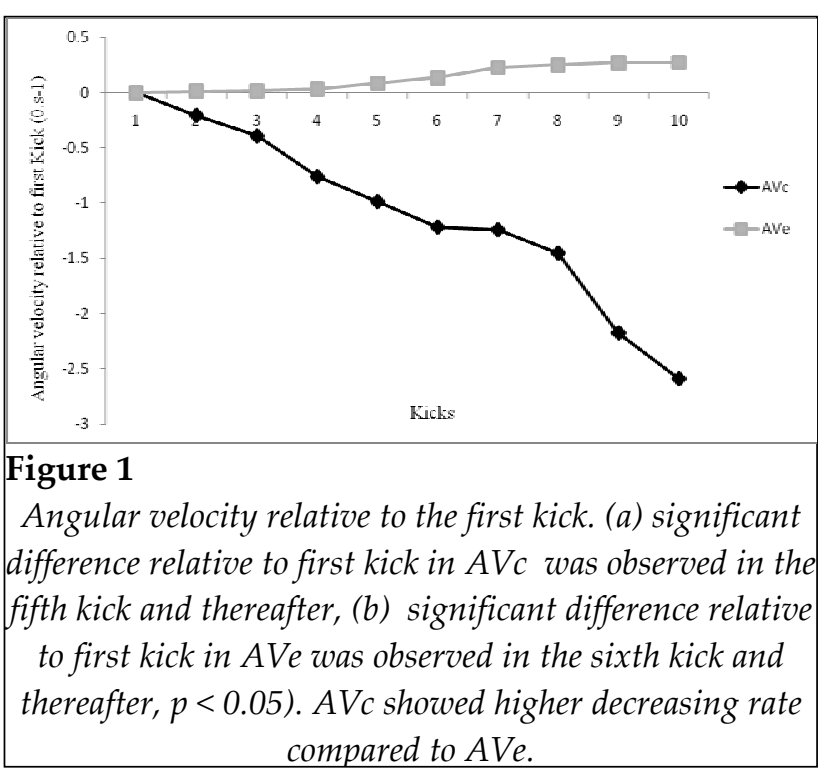

\section{Result}

The AVe result of the sixth kick, relative to the first kick $\left(0.13 \pm 0.02{ }^{\circ} . \mathrm{s}^{-1}\right)$, was significantly lower when compared to the other kicks $(\mathrm{p} \leq 0.01)$ (Figure 1). In addition, the AVc result of the fifth kick, relative to the first kick $\left(-0.98 \pm 0.35^{\circ} . \mathrm{s}^{-1}\right)$, was significantly lower when compared to other kicks (with $\mathrm{p} \leq$ 0.01) (Figure 1). As presented in Figure 2, the Te result of the fourth kick, relative to the first kick (0.002 $\pm 0.0002 \mathrm{sec})$, was significantly lower when compared to other kicks $(\mathrm{p} \leq 0.011)$ (Figure 1). Furthermore, the Tc result of the fifth kick, relative to the first kick $(0.006 \pm 0.0001 \mathrm{sec})$, was significantly lower when compared to other kicks (with $\mathrm{p} \leq 0.029$ ) (Figure 2).

\section{Discussion}

The main findings in this study were (1) significant increases in AVe after the $6^{\text {th }}$ kick, (2) significant decreasing in AVc after the $5^{\text {th }}$ kick, (3) significant increasing in Te after the $4^{\text {th }}$ kick, and (4) significant rise in Tc after the $5^{\text {th }}$ kick.

It seems that the most important reason for a decreasing velocity curve (Figure 1) during 10 consecutive instep kicks is the effect of fatigue on leg extensors, which are the main force producing muscle groups by SSC. Fatigue is a very complex phenomenon, and it may be described as a loss of force generating capability or an inability to sustain further exercise at the required level (Strojnik and Komi, 1998; Miyaguchi and Demura, 2008a). Present findings showed this effect of fatigue on recent variables, such as significant increases in AVe after the

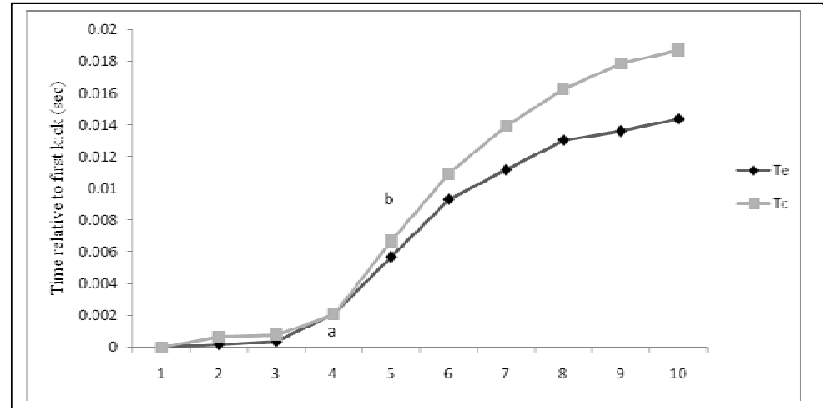

Figure 2

Time relative to the first kick. (a) significant difference relative to first kick in Te was observed in the fourth kick and thereafter, (b) significant difference relative to first kick in Tc was observed in the fifth and thereafter. Te shows relatively faster time to fatigue compared to Tc.

$6^{\text {th }}$ kick, relative to the first kick, however, there were significant decreases observed in $\mathrm{AV} c$ after the $5^{\text {th }}$ kick, relative to first kick. While eccentric contraction produces more force than concentric contraction, it appears that the effects of fatigue occur earlier with concentric contractions than eccentric contractions. Therefore, this affect influences $\mathrm{AVc}$ and Tc later, which is an indicator of strong SSC.

Utilization of SSC is characteristic for soccer instep kick, which is the main factor in producing more force and resulting in high ball velocity. In SSC, the action of the muscle during the eccentric phase influences the subsequent concentric phase (Miyaguchi and Demura, 2008b), therefore, AVc depends to AVe. These influences are expected to take place both in the elastic components of the muscle, as well as in the neural input. The series elastic component and its intracellular parts, as well as tendon and aponeurosis, constitute a major part of elastic structures of the tendomuscular system. The proprioceptive reflex systems and the central nervous system are required to adjust the motor programme to regulate the elastic potential (Dyhre-Poulsen et al., 1991), which is characteristic of SSC. It has been welldemonstrated that pre-stretching of an active muscle enhances its performance in subsequent concentric work. However, if the time interval between stretching and shortening (coupling time) is too long, the stored elastic energy can be wasted, and the mechanical efficiency of the concentric force will decrease (Kyrolainen and Komi, 1995). While elastic potential may result from many physiological or anatomical complexities, it seems that the ability of muscles to store and utilize elastic energy is dependent on stretching velocity and muscle length, the 
force attained at the end of the pre-stretch, as well as on the coupling time between the eccentric and concentric phases of the performance (Kyrolainen and Komi, 1995; Miyaguchi and Demura, 2008a; Miyaguchi and Demura, 2008b).

Therefore, it is clear that fatigue after 5 kicks effects leg extensors to contract concentrically, which result in an increase in Tc and subsequently, AVc. On the other hand, fatigue after 6 kicks appears within the extensor leg, which results in an increase in Te, followed by AVe. This change in SSC results in more reduction in angular velocity of the shank, thereafter. While many factors contribute to kicking success, the velocity of the ball is the main biomechanical indicator of success., In generating ball velocity, one of most important factors is the speed of the foot before impact, which depends on such variables as kinematics and kinetics of the thigh and shank (Dorge et al., 2002). We can summarize that a decreased shank angular velocity reduces foot speed, which ultimately results in a reduced ball velocity, compared to earlier kicks.

In summary, these results indicate that experienced adult male soccer players maintained high kinematic characteristics relative to SSC during the first 5 kicks. AVe showed significant increases after the sixth kick, relative to the first kick. In contrast,
AVc demonstrated a significant decrease after the 5th kick, relative to the first kick. Te increased significantly after the fourth kick, relative to the first kick, but Tc showed a significant increase after the fifth kick, relative to first kick. According to findings of this study, it seems that the transfer of energy between eccentric and concentric phases was drastically reduced during 10 consecutive soccer instep kicks.

Finally, we concluded that 5 consecutive kicks are adequate to achieve high kinematic responses related to SSC. This implies that 5 kick trials are optimal for players to perform a well-coordinated segmental pattern, and for researchers to select the best kick between these 5 kick trials for final analysis, because force production by SSC is obtained at a high level during first 5 kicks, in comparison to the second 5 kicks. The present results also suggest that coaches and trainers should design not more than five consecutive soccer instep kicks for their players during soccer training sessions. Furthermore, these results suggest strength and conditioning trainers use five consecutive instep kicks as the optimal number in designing their programs when they use soccer skill to improve players' fitness level.

\section{References}

Amiri-Khorasani, M., Osman, N.A.A., Yusof, A. Biomechanical responses of Instep kick between different positions in professional soccer players. J Hum Kinetics.2009; 22: 21-27.

Barfield, W. Effects of selected kinematic and kinetic variables on instep kicking with dominant and non dominant limbs. J Hum Move Studies. 1995; 29: 251-272.

Barlet, R. Sports Biomechanics: reducing injury and improving performance.E \& FN Spoon, an imprint of Routledge, London and New York. 2006.

Bates, B.T., Dufek, J.S., Davis, H.P. The effect of trial size on statistical power. Med Sci Sports Exerc. 1992; 24, 10591065.

Dorge, H., Bull-Andersen, T., Sorensen, H. Simonsen, E. Biomechanical differences in soccer kicking with the preferred and the non-preferred leg. J Sorts Sci. 2002; 20, 293-299.

Dyhre-Poulsen, P., Simonsen, E.B., Voigt, M. Dynamic control of muscle stiffness and H reflex modulation during hopping and jumping in man. J Physiology. 1991; 437:287-304.

James, C. R., Herman, J. A., Dufek, J. S., Bates, B. T. Number of trials necessary to achieve performance stability of selected ground reaction force variables during landing. J Sports Sci Med. 2007; 6, 126-134.

Kellis, E., Katis, A. Biomechanical characteristics and determinants of instep soccer kick. J Sports Sci Med. 2007; 6: 154-165. 
Kyrolainen, H., Komi. P.V. The Function of Neuromuscular System in Maximal Stretch-shortening Cycle Exercises: Comparison Between Power- and Endurance-trained Athletes. Journal of Electromyography and Kinesiology. 1995; 5, 15-25.

Lees, A., Nolan, L. The biomechanics of soccer: A review. J Sports Sci. 1998; 16: 211-234.

Levanon, J., Dapena, J. Comparison of the kinematics of the full-instep and pass kicks in soccer. Med Sci Sports Exerc. 30: 917-927, 1998.

Miyaguchi, K., Demura, S. Lateral dominance of stretch-shortening cycle performance in unilateral and lateral athletes. J Sports Med Physical Fitness. 2008a; 48(1), 24.

Miyaguchi K., Demura S. Relationships between Stretch-shortening Cycle Performance and Maximum Muscle Strength. J Strength Cond Res. 2008b; 22(1):19-24.

Manolopoulos, E., Papadopoulos, C., Salonikidis, K., Katartzi, E., Poluha, S. Strength training effects on physical conditioning and instep kick kinematics in young amateur soccer players during preseason. Percept Motor Skills. 2004; 99: 701-710.

Nunome, H., Asai, T., Ikegami, Y., Sakurai, S. Three-dimensional kinetic analysis of side-foot and instep soccer kicks. Med Sci Sports Exerc. 2002; 34: 2028-2036.

Nunome, H., Ikegami, Y., Kozakai, R., Apriantono, T., Sano, S. Segmental dynamics of soccer instep kicking with the preferred and non-preferred leg. J Sorts Sci. 2006; 24: 529-541.

Sedano-Campo, S., Vaeyens, R., Philippaerts, R.M., Redondo, J.C., De Benito, A.M., Cuadrado, C. Effects of lowerlimb plyometric training on body composition, explosive strength, and kicking speed in female soccer players. $J$ Strength Cond Res. 2009; 23(6): 1714-1722.

Strojnik, V., Komi, P.V. Neuromuscular fatigue after maximal stretch-shortening cycle exercise. Journal of Applied Physiology. 1998; 84(1): 344-350.

\section{Acknowledgements}

The authors would like to thank football players for their kind participation and motion analysis lab technicians. This work was supported by the University Malay Research Grant.

\section{Corresponding author}

\section{Mohammadtaghi Amiri-Khorasani}

Sport Centre, University of Malaya, 50603, Kuala Lumpur, Malaysia

Phone/Fax. 0060126034134

E-mail: amirikhorasani@gmail.com 